\title{
Domestic Short Hair Cat
}

National Cancer Institute

\section{Source}

National Cancer Institute. Domestic Short Hair Cat. NCI Thesaurus. Code C77124.

The name for a short-haired cat that does not have a pedigree nor belong to a specific cat breed. The domestic short-hair cat is a small carnivorous crepuscular mammal that has short fur length and is more slender and active than the domestic long-haired cat. It may have varied coat length and coat color in a single or multi-colored pattern. The domestic short-hair cat has a flexible, compact body and long tail that enhances balance, as well as keen eyesight and retractable claws. The female cat exhibits high fecundity; sexual maturation in the female occurs between 6-12 months of age, producing up to 3 litters of 4-8 kittens per year. Male domestic long-hair cats become sexually mature at 922 months. The gestational period of the cat embryo is 63-65 days and the average lifespan of a domestic short haired cat is 10-15 years. The domestic short-hair cat is used most commonly in medical research involving viral pathogenesis and vaccination studies. 